\title{
Data Pre-Processing/Model Initialisation in Neurofuzzy Modelling of Structure-Property Relationships in Al-Zn-Mg-Cu Alloys
}

\author{
O. P. FEMMINELLA, ${ }^{1,2)}$ M. J. STARINK, ${ }^{1)}$ M. BROWN, ${ }^{3)}$ I. SINCLAIR, ${ }^{1)}$ C. J. HARRIS ${ }^{2)}$ and \\ P. A. S. REED ${ }^{11}$ \\ 1) Department of Engineering Materials, University of Southampton, Southampton S017 1BJ, UK. \\ 2) Department of Electronics and Computer Science, University of Southampton, Southampton S017 1BJ, UK. \\ 3) Formerly at University of Southampton, now at Unilever Research, Port Sunlight, Wirral, UK.
}

(Received on March 25. 1999; accepted in final form on July 12, 1999)

\begin{abstract}
The paper deals with the application of multiple linear regression and neurofuzzy modelling approaches to $7 x x x$ series based aluminium alloys. 36 compositional and ageing time variants and subsequent proof strength and electrical conductivity measurements have been studied. The input datasets have been transformed in two ways: to reveal more explicit microstructural information and to reflect some empirical findings in the literature. Neurofuzzy modelling exhibited improved performance in modelling proof strength and electrical conductivity cf. the multiple linear regression approach. Electrical conductivity is best modelled using the explicit microstructural input dataset, whilst proof strength is best modelled by a further modification of this dataset, decided upon after inspection of the subnetwork structures produced by neurofuzzy modelling. Neurofuzzy modelling offers a transparent empirically based data-driven approach that can be combined with pre-processing of the data and initialising of the model structure based upon physical understanding. An iterative modelling approach is defined whereby data-driven empirical modelling approaches are first used to assess underlying data structures and are validated against physically based understanding, these then inform subsequent initialised neurofuzzy models and input data transformations to provide both optimal subset and feature representation.
\end{abstract}

KEY WORDS: neural networks; fuzzy logic; microstructure; $7 x x x$ alloys; electrical conductivity.

\section{Introduction}

The use of Artificial Neural Networks (ANN) for intelligent data analysis (trend and clustering discovery) is attracting a significant amount of interest over a wide range of industrial sectors. ${ }^{1,2)}$ These provide powerful empirical tools for modelling and classifying non-linear, interdependent, and noisy data sets that are not fully understood or are not amenable to exact analytical solutions. Probably the main reason for their widespread use is that the underlying relationships do not have to be explicitly specified, rather these are implicitly learnt from the data, and hence ANNs may significantly outperform conventional data regression analysis when prior problem knowledge is poor. ${ }^{2)}$ One significant drawback however, is the lack of transparency in the modelling process, and this has hindered more widespread use of the techniques. Generally, neural networks are difficult to validate and have little relationship to conventional physical models, although significant research effort has been directed recently towards incorporating existing physical knowledge into these systems and extracting rules. The neurofuzzy (NF) data modelling algorithms used in this work combine mathematically rigorous non-linear regression-type networks (based on an additive spline representation) with an explanation facility that is based on fuzzy logic, providing a means for combining empirical data with established expert knowledge in both building and validating the model. ${ }^{1)} \mathrm{A}$ recent successful NF application concerned Ni-based superalloy fatigue behaviour, which demonstrated that a combination of basic materials' properties and test conditions readily provided physically reasonable models of near-threshold crack growth. ${ }^{3)}$ Pure neural network models of equivalent data frequently exhibited unrealistic physical characteristics, engendering extensive model interrogation and verification processes. ${ }^{4)}$ Successful classical neural network modelling of complex materials characteristics has been reported in the literature ${ }^{5-7)}$ e.g. phase transformation and mechanical property prediction for steels. ${ }^{6,7)}$ This paper describes a critical progression in the effective use of such methods via the ability to include, $a b$ initio, known physical principles within sophisticated neural network data modelling frameworks, and enables direct assessment of the physical relationships within the transparent models produced.

The dataset considered in the present work contains data on the properties of various $\mathrm{Al}-\mathrm{Zn}-\mathrm{Mg}-\mathrm{Cu}-\mathrm{Zr}$ based alloys. High strength $7 x x x(\mathrm{Al}-\mathrm{Zn}-\mathrm{Mg}-\mathrm{Cu})$ alloys make up the largest volume of aluminium alloy sold to the aerospace industry and due to the ever increasing demands for property improvement most research and development work of aluminium producers is directed 
towards this alloy system. $7 x x x$ alloys have 3 critical target properties: yield strength, toughness and stress corrosion cracking (SCC) resistance. SCC is hard to measure on production-line time-scales so generally the more easily obtainable electrical conductivity is used as a measure of SCC resistance. Of the three main properties, yield strength and electrical conductivity are determined mainly by the precipitation processes that occur during commercial thermal treatments of the alloys. The third property, toughness, is a complex function of matrix flow characteristics, intermetallic particle populations (coarse primary constituents and dispersoids), grain structure and coarse heterogeneous precipitation (particularly on boundaries and dispersoids). The balance between the three main properties of $7 x x x$ alloys is a precarious one, with compositional and processing parameters having conflicting effects on the various properties. Modelling of the properties is hampered by the complexity of the relationship between primary process variables (composition, quenching rate, ageing time, ageing temperature, etc.) and target properties, especially toughness and strength. Thus, data for this alloy system is especially suited for investigation using adaptive numerical modelling. Optimal feature representation (data pre-processing) using knowledge of precipitation processes in these alloys may be an effective means of enhancing the predictive capability of models.

\section{Dataset}

The present, proprietary dataset comprises the results from heat treatment trials carried out by DERA, Farnborough, U.K., under contract from British Aluminium Plate, on a range of alloy compositions that broadly cover the high strength variants of the $7 x x x$ series aluminium alloys. Although of limited size, the dataset reflects its experimentally designed origin as the input distributions were wide ranging if somewhat sparse. For each alloy, composition levels of $\mathrm{Zn}\left(x_{\mathrm{Zn}, \mathrm{w}}\right), \mathrm{Mg}$ $\left(x_{\mathrm{Mg}, \mathrm{w}}\right), \mathrm{Cu}\left(x_{\mathrm{Cu}, \mathrm{w}}\right), \mathrm{Zr}\left(x_{\mathrm{Zr}, \mathrm{w}}\right), \mathrm{Fe}\left(x_{\mathrm{Fe}, \mathrm{w}}\right)$ and $\mathrm{Si}\left(x_{\mathrm{Si}, \mathrm{w}}\right)$ in $\mathrm{wt} \%$ have been determined. The alloys were solution treated and subsequently aged for various times at a single temperature which is similar to the ones used for commercial $\mathrm{T} 7$ tempers. The subsequent $0.2 \%$ proof stress $\left(\sigma_{0.2}\right)$ and electrical conductivity $\left(\sigma_{\mathrm{el}}\right)$ have been measured for each alloy variant. 36 composition/ageing time variants have been studied, and as a result this dataset can be considered relatively small for the successful application of ANN type approaches. For each differing condition a number of experimental tests had been carried out, mean property measurements have been used throughout.

The inputs (and outputs) were transformed to have a zero mean and unit variance. This simple form of preprocessing is beneficial as it removes non-essential sources of ill-conditioning, improving the condition of the dataset and allowing comparison of magnitudes of the weights determined in the multiple linear regression (MLR). To preserve commercial confidentiality, all data involving input variables has been presented in this normalised form, final output (property) values and mean squared error (MSE) values are however presented in un-normalised form. In the NF modelling framework, in order to facilitate model refinements, which require exact specification of locations (knot placements) along the fuzzified input variables, the input variables are transformed to lie within \pm 1 .

\section{Empirical Data Modelling}

\subsection{Neurofuzzy Data Modelling}

Fuzzy logic was developed as a way of modelling natural language explanations used by human experts. Fuzzy algorithms have been applied to a wide range of complex engineering applications, where the possibility for fast system development is exploited. In such applications, the systems store information as a set of easily interpreted, linguistically based fuzzy rules, for example one can define set definitions for natural language terms such as short, medium and long and then interpolate between these rules. However, there are a number of different implementation methodologies, and there is a lack of rigorous theory to explain how the systems generalise and to describe the relative advantages of different systems. Fuzzy sets form the basis of fuzzy logic and they provide absolute mathematical interpretation to vague concepts. ${ }^{8)}$ In contrast to classical Boolean logic an element may be a partial member of a fuzzy set, e.g. if the ageing time is $6 \mathrm{hrs}$, the membership of the set of long might be taken to be: $\mu_{\text {long }}$ (ageing time $)=0.8$. So there is a gradual transition between membership and non-membership whereas in classical logic an element must be wholly included or entirely excluded from a set (i.e. ageing time is either long or not long). A fuzzy rule is of the form:

$$
\begin{aligned}
r_{i j}: & \operatorname{IF}\left(x_{1} \text { is } A_{1}^{i} \text { AND } x_{2} \text { is } A_{2}^{i}\right. \\
& \left.\operatorname{AND} \cdots \text { AND } x_{n} \text { is } A_{n}^{i}\right) \text { THEN }\left(y \text { is } B^{j}\right) c_{i j}
\end{aligned}
$$

Where $x_{k}$ is the $k^{\text {th }}$ real-valued input, $y$ is the output, $r_{i j}$ is the $i j^{\text {th }}$ fuzzy rule, $A_{i}^{k}$ is the univariate linguistic term (or fuzzy set) and $B^{j}$ is the corresponding output linguistic rule.

\section{E.g.: IF (Cu is high) AND (ageing time is medium) THEN (proof stress is high)}

Associated with each rule is a rule confidence, $c_{i j}$, which is a measure of the degree of the contribution of the rule to the output. A rule confidence of zero means that the rule does not contribute to the output, and a rule confidence of one means that the rule is completely true. The set of fuzzy sets used to represent an input, and the functions used to express these sets, may be pictorially represented to allow easy interpretation. A simple example of this, with three triangular (second order) fuzzy sets and a variable (e.g. ageing time) with value range $0-10$ is shown in Fig. 1 . At any given value of the variable, membership of all the possible sets adds to unity. For example, at a value of 2.5 the membership of the set short is 0.5 , of the set medium is 0.5 and of the set long is zero. The point at which the membership of two fuzzy sets comes to zero (at a value of 0.5 in this case) is called a knot, and is likely to represent a change 


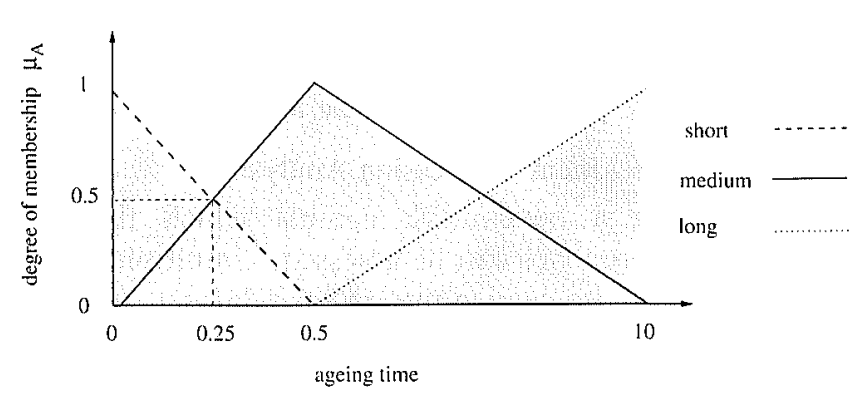

Fig. 1. Schematic representation of a fuzzy variable.

in the trend between input and output. The actual shape of the trend between input and output is determined by the rule confidence.

Neurofuzzy modelling combines such qualitative, rule-based representation of the derived model with the structural and learning abilities commonly associated with ANNs. The modelling abilities can be assessed, the structure analysed and standard algorithms can be used to train weights and investigate various structural configurations within a model hypothesis testing framework. ${ }^{9)}$ The NF modelling employs an analysis of variance (ANOVA) representation to model the additive structural relationships that may exist in the data. This allows the network's output (predicted properties) to be expressed as a sum of a number of small NF systems (or subnetworks), each with a limited number of inputs (e.g. compositional variables, ageing time) from the main input vector. The output is given by:

$$
\begin{aligned}
& y(\mathbf{x})=\sum_{i=1}^{p} \mu_{A^{i}}(\mathbf{x}) \sum_{j=1}^{q} c_{i j} y_{j}^{c}=\sum_{i=1}^{p} \mu_{A^{i}}(\mathbf{x}) w_{i} \\
& =\sum_{i=1}^{p} a_{i}(\mathbf{x}) w_{i}=\sum_{i=1}^{s} s_{i}\left(\mathbf{x}_{i}\right)
\end{aligned}
$$

where $\mu_{A}^{i}(\mathbf{x})$ is the $i^{\text {th }}$ multivariate fuzzy membership function generated by fuzzy intersection of the linguistic variables $A_{i}^{k}, y_{j}^{c}$ is the centre of the $j^{\text {th }}$ fuzzy output set and $w_{i}$ is the weight associated with the corresponding membership function. This representation is identical to a B-spline network, where the multivariate basis functions, $a_{i}(\mathbf{x})$, are the multivariate fuzzy membership functions. Output $s_{i}\left(\mathbf{x}_{i}\right)$ is the output from the $i^{\text {th }}$ subnetwork whose input vector $\mathbf{x}_{i}$ is a smaller subset of the total input vector $\mathbf{x}$. The structure of this type of network is shown in Fig. 2. Each subnetwork is implemented as a conventional NF model, where the output is formed from a linear combination of fuzzy input basis functions which are implemented as B-spline piecewise polynomial basis functions. This simplified additive network reduces the resources (quantity of data) required to implement a robust fuzzy system (compared to one large network taking all the input variables), and gives improved generalisation ability, while also increasing the transparency of the network by simplifying the linguistic fuzzy rules produced. ${ }^{10)}$ In addition, when the subnetworks each have a small number of inputs ( 1 or 2), the additive structure can reveal simple trends in the network's output by enabling visualisation of the output of each subnetwork. This is especially important

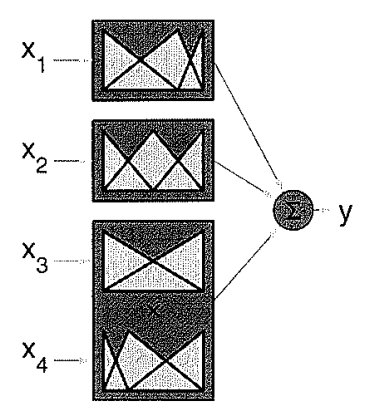

Fig. 2. Representation of a NF model structure.

for verification and validation as this can be compared to simple linear models and can highlight regions of differing trends that can be verified against expert knowledge. The NF framework allows the designer to use their own expertise to formulate rules to initialise the network and also to verify relationships extracted from the data by the network against their current physical understanding of the system. This is in contrast to the physically ambiguous character of pure neural networks, which can be seen as "black-box" learning systems.

\subsection{Model Construction and Training Neurofuzzy Networks}

Given a particular NF network structure, the weights (in effect the rules) can be estimated using robust linear optimisation algorithms, hence the construction procedure is a search for an appropriate model structure. For a given NF model, possible refinements to construct the model are:

- Univariate addition: inclusion of an input by including another additive subnetwork;

- Tensor product: inclusion of an input to an existing subnetwork, allowing input variable interactions;

- Knot insertion: the flexibility of a subnetwork is increased by refining the rulebase through introduction of a new basis function;

- Subnetwork deletion: an existing subnetwork is removed;

- Tensor splitting: a subnetwork with $n \geq 2$ inputs is replaced by one that depends on less combinations of the $n$ inputs;

- Knot deletion: the flexibility of a subnetwork is reduced by reducing the number of basis functions;

- Reduce order: the order of basis functions in a subnetwork is reduced.

These refinements are combined, to provide a coherent model search, into a forward selection-backward elimination (FS/BE) pass structure, in which initially the overall model structure is identified by a set of model building passes and subsequent model pruning passes are employed to remove any redundant sources of variance, to give the most parsimonious model. Parsimonious models are desirable as each redundant input variable in a network increases its complexity without adding any useful information to the model. Typically an ANN will use mean squared error (MSE) as a measure of prediction performance, where $\mathrm{MSE}=(1 / N) \sum_{i}^{N}\left(t_{i}-y_{i}\right)^{2} . N$ is the number of data pairs in the set, $t_{i}$ is the target value (i.e. 
the measured output) and $y_{i}$ is the predicted output. In NF data modelling statistical significance measures are used to balance the network's MSE against its size and the amount of available data. This ensures that an acceptable level of performance is achieved with respect to the network's size and the quantity of the available training data. ${ }^{9)}$ The network construction algorithms are stopped when the statistical significance measure starts to rise. To overcome problems associated with finding local minima in MSE and statistical significance, model search termination criteria were appropriately set. ${ }^{11)}$ In light of empirical results ${ }^{11)}$ the structural risk minimisation $(\mathrm{SRM})^{12)}$ principle was adopted as the statistical significance measure. Due to the small sample size, the order of the B-splines considered was limited to be $\leq 2$, to prevent the inclusion of severely ill-conditioned basis functions.

For a given set of inputs and corresponding output(s) the network can be trained in either of the following ways:

- the training data are presented to an initially empty model and the automatic model construction algorithms search for the network structure;

- the data can be presented to an initial network structure which reflects prior knowledge and system understanding and network construction algorithms used to refine the model structure;

- connections and rule bases (model structure) can be defined and the network left to determine only the weights/rule confidences.

Initially the first method of training was used in order to investigate what structures the network could identify from the raw data (dataset A). Pre-processing of the input data was then carried out in the light of both physical metallurgy understanding (dataset B) and rule-of-thumb approximations reported in the literature (dataset C) which are detailed in later sections of the paper. The network was then presented with these two revised datasets to examine to what degree the incorporation of "expert knowledge" at the data preprocessing stage would improve the modelling performance. All data available was used in the model construction to retain the maximum possible information. Models constructed were assessed in terms of their statistical significance measure (SS), an adjusted training set MSE taking into account the number of parameters $\left(n_{w}\right)$ fitted in the model. Once the model was identified, a leave-one-out cross-validation (LOOCV) strategy was employed to assess the generalisation performance of the model, by estimating the model's prediction error. Since model identification was determined using all the data available, the LOOCV estimate of prediction error will still exhibit some bias. Target output (measured properties) versus model output (predictions) were also examined. Relationships identified were validated against known physical metallurgy principles.

From examination of the models identified by the automatic model construction algorithms and using $a$ priori knowledge of the likely physical processes and trends characterising alloy behaviour, model initialisation was investigated, by specifying a prior functional form for the model (i.e. specifying a set of fuzzy rules).
The three datasets (A, B and C) were then presented to this predetermined model structure so that the efficacy of the data pre-processing could be assessed further.

\subsection{Multiple Linear Regression Analysis}

To provide a benchmark against which the NF modelling approaches can be assessed, a multiple linear regression was also carried out on each dataset. A linear model is given by equation (3):

$$
y(x)=w_{0}+w_{1} x_{1}+w_{2} x_{2}+\cdots+x_{\mathrm{p}} x_{\mathrm{p}}
$$

where $\mathbf{x}=\left[x_{1}, \cdots, x_{\mathrm{p}}\right]^{\mathrm{T}}$ is the model's input vector, $w_{1}, \cdots, w_{\mathrm{p}}$ are unknown fitting parameters to be estimated, $w_{0}$ is an unknown bias term and $y$ is the predicted output. The unknown vector of parameters, $w_{p}$, can be estimated in the least squares sense.

The next section of the paper details and justifies the two data pre-processing approaches adopted to further improve the modelling process.

\section{Transformation of Input Variables}

\subsection{Precipitation in Al-Zn-Mg-Cu Alloys}

A large body of work on the thermodynamics, microstructure and microstructure-property relations of $7 x x x(\mathrm{Al}-\mathrm{Zn}-\mathrm{Cu}-\mathrm{Mg})$ type alloys exists. ${ }^{13-20)}$ Using this knowledge, modelling of the strength of ternary $\mathrm{Al}-\mathrm{Zn}-\mathrm{Mg}$ alloys on an analytical microstructure related basis $^{21)}$ has been carried out, and modelling of the quaternary $7 x x x$ alloys on the basis of similar microstructure-property relations appears to be possible. ${ }^{20)}$ Instead of modelling properties using either purely adaptive numerical modelling approaches or purely analytical microstructure-property models, it may be advantageous to use the physical modelling to aid datadriven modelling approaches. In the present work, the original raw input data for the present $\mathrm{Al}-\mathrm{Zn}-\mathrm{Cu}-$ $\mathrm{Mg}-\mathrm{Zr}$ type alloys have been transformed into data that reflect key microstructural features, most notably the amounts of the main phases. In the interest of clarity, we have limited ourselves to considering just two microstructural elements: the volume fraction of the main strengthening phase $\eta^{\prime}$ that forms in the alloys and the volume fraction of the main coarse intermetallic phase: the $\mathrm{S}\left(\mathrm{Al}_{2} \mathrm{MgCu}\right)$ phase.

\subsection{Main Microstructure Related Strengthening and Conductivity Effects}

During homogenising and solution treatments some of the $\mathrm{Cu}$ and $\mathrm{Mg}$ present in the $7 x x x$ alloys will not dissolve in the Al-rich matrix because some $\mathrm{S}\left(\mathrm{Al}_{2} \mathrm{MgCu}\right)$ phase will be stable at the solution treatment temperature. The $\mathrm{Cu}$ and $\mathrm{Mg}$ "tied-up" in the S phase will thus not cause any decrease in $\sigma_{\mathrm{el}}$ of the Al-rich phase (the Al-rich phase is the only significant conductive pathway in the alloys) and, further, are not available for subsequent precipitation hardening during ageing (precipitation hardening is the dominating strengthening mechanism). This means that, in first approximation, the $\mathrm{Cu}$ and $\mathrm{Mg}$ present in $\mathrm{S}$ phase have become largely irrelevant in affecting the $\sigma_{\mathrm{el}}$ and $\sigma_{0.2}$ of the alloy. (However, the $S$ phase will have a detrimental effect on a third important 
mechanical property: the toughness.) Thus, modelling of $\sigma_{\mathrm{cl}}$ and $\sigma_{0.2}$ of the alloy variants may be improved by transforming the atomic fraction of $\mathrm{Cu}$ and $\mathrm{Mg}, x_{\mathrm{Cu}}$ and $x_{\mathrm{Mg}}$ into $x_{\mathrm{Cu}, \alpha}$ and $x_{\mathrm{Mg}, \alpha}$, the atomic fraction of $\mathrm{Cu}$ and $\mathrm{Mg}$ dissolvable in the Al-rich $(\alpha)$ phase. The latter quantities can be obtained as follows.

If the stoichiometry of a phase is fixed, the solubility of an intermetallic phase can often be described by the regular solution model. ${ }^{13,22,23)}$ In this model the solvus related to an intermetallic phase $\mathrm{M}_{m} \mathrm{~A}_{\mathrm{a}} \mathrm{B}_{\mathrm{b}} \mathrm{C}_{\mathrm{c}}$ (M is the main constituent of the alloy, and $\mathrm{A}, \mathrm{B}, \mathrm{C}$ are the alloying elements) is given by:

$$
\left(c_{\mathrm{A}}\right)^{a}\left(c_{\mathrm{B}}\right)^{b}\left(c_{\mathrm{C}}\right)^{c}=c_{\mathrm{o}} \exp \left[\frac{-\Delta H_{\mathrm{sol}}}{k_{\mathrm{B}} T}\right]
$$

where $\Delta H_{\text {sol }}$ is the enthalpy of formation of one 'molecule' of $\mathrm{M}_{m} \mathrm{~A}_{a} \mathrm{~B}_{b} \mathrm{C}_{c}, k_{\mathrm{B}}$ is Boltzmann's constant and $c_{\mathrm{o}}$ is a constant. If appropriate values for $\Delta H_{\mathrm{sol}}, c_{0}, a, b$ and $c$ for each phase can be derived from available solubility data, a phase diagram can be constructed. However, only for $T=460^{\circ} \mathrm{C}$ significant data on the solvi of all phases are available. ${ }^{16)}$ For the $\mathrm{S}$ phase the $\Delta H_{\mathrm{sol}}(\mathrm{S})$ in ternary alloys has been determined previously, ${ }^{23)}$ and by combining solvus data at $460^{\circ} \mathrm{C}^{16)}$ with $\Delta H_{\text {sol }}$ the $\mathrm{S}$ solvus as a function of the temperature can be estimated. For $\mathrm{S}$ phase, at the solution treatment temperature applied to the present alloys, it is thus estimated:

$$
\left(c_{\mathrm{Mg}}\right)\left(c_{\mathrm{Cu}}\right)=2.96 \times 10^{-4}
$$

$x_{\mathrm{Cu}, \alpha}$ and $x_{\mathrm{Mg}, \alpha}$ can then be calculated as follows:

$$
\begin{aligned}
& \text { if }\left(x_{\mathrm{Mg}}\right)\left(x_{\mathrm{Cu}}\right) \leq 2.96 \times 10^{-4} \text { then } x_{\mathrm{Cu}, \alpha}=x_{\mathrm{Cu}} \\
& \text { and } \quad x_{\mathrm{Mg}, \alpha}=x_{\mathrm{Mg}} \\
& \text { if }\left(x_{\mathrm{Mg}}\right)\left(x_{\mathrm{Cu}}\right)>2.96 \times 10^{-4} \\
& \text { then } x_{\mathrm{Cu}, \alpha}=-\frac{1}{2}\left(x_{\mathrm{Mg}}-x_{\mathrm{Cu}}\right) \\
& +\frac{1}{2} \sqrt{\left(x_{\mathrm{Mg}}-x_{\mathrm{Cu}}\right)^{2}+4 \times 2.96 \times 10^{-4}}
\end{aligned}
$$$$
\text { and } x_{\mathrm{Mg}, \alpha}=x_{\mathrm{Mg}}-\left(x_{\mathrm{Cu}}-x_{\mathrm{Cu}, \alpha}\right)
$$

and the atomic fraction of $\mathrm{S}$ phase, $x_{\mathrm{S}}$, (i.e. the number of atoms present as $\mathrm{S}$ phase divided by the total number of atoms in the system) is given by:

$$
x_{\mathrm{s}}=4\left(x_{\mathrm{Cu}}-x_{\mathrm{Cu}, \alpha}\right)
$$

Whilst several precipitation sequences in $7 x x x$ alloys have been reported, it is well established that the main sequence responsible for most of the age-hardening in $7 x x x$ alloys is:

$$
\operatorname{sss} \alpha \rightarrow \text { GP zones } \rightarrow \eta^{\prime} \rightarrow \eta
$$

where $\operatorname{sss} \alpha$ is the supersaturated solid solution of the Al-rich $(\alpha)$ phase, $\eta^{\prime}$ is thought to have a stoichiometry close to $\mathrm{Mg}_{4} \mathrm{Zn}_{11} \mathrm{Al}^{24)}$ whilst $\eta$ is based on $\mathrm{MgZn}_{2} \cdot{ }^{14,24)}$ It is known that in the peak aged condition most of the GP zones formed early on in the precipitation sequence are replaced by $\eta^{\prime}$ phase, whilst $\eta$ phase (if at all present in the slightly overaged alloys investigated) will not cause significant strengthening. As a result $\eta^{\prime}$ will be the main hardening phase. The maximum atomic fraction of $\eta^{\prime}$ that can form is given by:

$$
x_{\eta^{\prime}}=16 \min \left(\frac{x_{\mathrm{Zn}}}{11}, \frac{x_{\mathrm{Mg}, \alpha}}{4}\right)
$$

It is thought that for the modelling of $\sigma_{0.2}$, the main mechanisms involved are precipitation hardening due to $\eta^{\prime}$ and solution hardening of the $\alpha$ phase due to $\mathrm{Zn}, \mathrm{Cu}$ and $\mathrm{Mg}$. In the latter mechanism $\mathrm{Mg}$ is expected to have the strongest influence, as solution strengthening due to $\mathrm{Mg}$ is generally considered to be more important than that due to $\mathrm{Cu}$ or $\mathrm{Zn} .{ }^{25,26)}$ The maximum level of precipitation hardening is expected to be determined by $x_{\eta^{\prime}}$ and the maximum level of solution hardening is mainly determined by $x_{\mathrm{Mg}, \alpha}$. The relative contributions of solution hardening and precipitation hardening will change with time as precipitation of alloying elements progresses, with precipitation hardening gradually replacing solution hardening as the main strengthening mechanism. For the present, overaged alloys, significant $\eta^{\prime}$ precipitation will have occurred in all alloys, and thus the main variables are thought to be (in order of importance) $x_{\eta^{\prime}}$, ageing time $(t), x_{\mathrm{Mg}, \alpha}$ and $x_{\mathrm{Cu}, \alpha}$.

It is further known that the amount of $\mathrm{Mg}$ that is left in solution after completed formation of the main precipitate(s) is an important variable in determining the properties of overaged alloys. ${ }^{27)}$ We have calculated this so-called "excess $\mathrm{Mg}$ ", $x_{\mathrm{Mg}, \mathrm{ss}}$, using a novel expression, which is, at present, proprietary. Also $x_{\eta^{\prime}}, x_{\mathrm{Cu}, \alpha}$ and $x_{\mathrm{Mg}, \alpha}$ are expected to have an influence with the $\mathrm{Zn}$ content related $x_{\eta}$, variable having a stronger effect than $x_{\mathrm{Cu}, \alpha}$ and $x_{\mathrm{Mg}, \alpha}{ }^{13)}$

From the above, it follows that the main variables determining $\sigma_{0.2}$ are $x_{\eta^{\prime}}$ and $t$, with secondary effects determined by $x_{\mathrm{Mg}, \alpha}$ and $x_{\mathrm{Cu}, \alpha}$. The main variables determining $\sigma_{\mathrm{el}}$ are $x_{\mathrm{Mg}, \mathrm{xs}}$ and $t$ with further minor contributions from $x_{\mathrm{Cu}, \alpha}$ and a very small influence due to $x_{\mathrm{Zn}}$ (or $x_{\eta^{\prime}}$ ). Thus in the modelling approaches described below we have applied these insights to construct a new set of input variables relevant to the properties to be modelled (dataset B) consisting of: $x_{\eta^{\prime}}$, $t, x_{\mathrm{Mg}, \alpha}, x_{\mathrm{Cu}, \alpha}, x_{\mathrm{Mg}, \mathrm{xs}}$ and $x_{\mathrm{s}}$.

\subsection{Rule of Thumb Variables: Ratios and Sums}

In technical publications alloys are often classified by sums and ratios of the weight percentages of the main alloying elements, and for $\mathrm{Al}-\mathrm{Zn}-\mathrm{Mg}-\mathrm{Cu}$ alloys some of the variables employed are $x_{\mathrm{Zn}, \mathrm{w}}: x_{\mathrm{Mg}, \mathrm{w}},\left(x_{\mathrm{Zn}, \mathrm{w}}+x_{\mathrm{Cu}, \mathrm{w}}\right)$ : $x_{\mathrm{Mg}, \mathrm{w}}$ and $x_{\mathrm{Zn}, \mathrm{w}}+x_{\mathrm{Mg}, \mathrm{w}}$. These quantities can have physical meanings, e.g. the $x_{\mathrm{Zn}, \mathrm{w}}: x_{\mathrm{Mg}, \mathrm{w}}$ ratio will exert an important influence on the balance of the main precipitation sequences operating in the alloy, but, in terms of solid state reactions, the relevance of adding weight percentages of atoms is, in general, unclear. However, it can be seen that with the atomic weight of $\mathrm{Zn}$ being about 2.7 times that of $\mathrm{Mg}$, adding $x_{\mathrm{Zn}, \mathrm{w}}+x_{\mathrm{Mg}, \mathrm{w}}$ (in weight percentage) may be some measure of the amount of strengthening $\eta^{\prime}$ phase, provided this phase has a broad range of stability around its central composition of $\mathrm{Mg}_{4} \mathrm{Zn}_{11} \mathrm{Al}$. In order to fully investigate possible permutations of input variables we have con- 
Table 1. Linear regression analysis of the data on $\sigma_{0.2}$ and $\sigma_{\mathrm{e} 1}$ employing three different sets of input data: the raw input data (dataset $\mathrm{A}$ ), the set transformed using microstructure related assessments (dataset B) and the set transformed using "rule-of-thumb" sums and ratios (dataset $\mathrm{C}$ ).

\begin{tabular}{|c|c|c|c|c|c|c|c|c|}
\hline \multicolumn{3}{|c|}{$\begin{array}{l}\text { Dataset } A \\
\text { (raw data) }\end{array}$} & \multicolumn{3}{|c|}{$\begin{array}{l}\text { Dataset B } \\
\text { (microstructure related } \\
\text { data) }\end{array}$} & \multicolumn{3}{|c|}{$\begin{array}{l}\text { Dataset C } \\
\text { ("rule-of-thumb" data) }\end{array}$} \\
\hline \multirow[t]{2}{*}{ Inputs } & \multicolumn{2}{|c|}{ Weights } & \multirow[t]{2}{*}{ Inputs } & \multicolumn{2}{|c|}{ Weights } & \multirow[t]{2}{*}{ Inputs } & \multicolumn{2}{|c|}{ Weights } \\
\hline & $\sigma_{0.2}$ & $\sigma_{\mathrm{ej}}$ & & $\sigma_{0.2}$ & $\sigma_{\varepsilon \mid}$ & & $\sigma_{0.2}$ & $\sigma_{\mathrm{e} \mid}$ \\
\hline \multirow[t]{2}{*}{$\mathrm{x}_{\mathrm{Zn}, w}$} & +0.43 & +0.04 & $x_{\eta^{\prime}}$ & +0.36 & -1.24 & $\mathrm{x}_{\mathrm{Zn}, \mathrm{w}}: \mathrm{x}_{\mathrm{Mg}, w}$ & -1.21 & +0.93 \\
\hline & & & $\mathrm{x}_{\mathrm{s}}$ & $+1 E^{-4}$ & +0.16 & $x_{Z n, w}+x_{M g, w}$ & +0.68 & -0.35 \\
\hline \multirow[t]{2}{*}{$\overline{\mathrm{x}_{\mathrm{Mg}_{\mathrm{g}, w}}}$} & +0.60 & -0.72 & $\mathrm{X}_{\mathrm{Mg, \alpha}}$ & +0.72 & +4.62 & $\left(\mathrm{x}_{\mathrm{Zn}, \mathrm{w}}+\mathrm{x}_{\mathrm{Cu}, \mathrm{w}}\right): \mathrm{x}_{\mathrm{Mg}, w}$ & +0.80 & -0.10 \\
\hline & & & $\mathrm{x}_{\mathrm{Mg}, \mathrm{xs}}$ & -0.06 & -5.99 & & & \\
\hline $\mathrm{XCu}, w_{1}$ & +0.26 & -0.05 & $\mathrm{x}_{\mathrm{Cu}, \alpha}$ & +0.26 & -0.15 & & & \\
\hline$\overline{\mathrm{x}_{\mathrm{Z}, w}}$ & +0.17 & -0.20 & & & & & & \\
\hline $\mathrm{X}_{\mathrm{Fc}, \mathrm{w}}$ & -0.13 & +0.12 & & & & & & \\
\hline $\mathrm{x}_{\mathrm{Si}, w}$ & +0.10 & -0.19 & & & & & & \\
\hline$t$ & -0.73 & +0.75 & $t$ & -0.73 & +0.75 & $t$ & -0.73 & +0.73 \\
\hline MSE & 642 & 3.48 & MSE & 629 & 3.27 & MSE & 602 & 3.52 \\
\hline
\end{tabular}

sidered the three sums and ratios $x_{\mathrm{Zn}, \mathrm{w}}: x_{\mathrm{Mg}, \mathrm{w}},\left(x_{\mathrm{Zn}, \mathrm{w}}+\right.$ $\left.x_{\mathrm{Cu}, \mathrm{w}}\right): x_{\mathrm{Mg}, \mathrm{w}}$ and $x_{\mathrm{Zn}, \mathrm{w}}+x_{\mathrm{Mg}, \mathrm{w}}$, in addition to ageing time, $t$. These four input variables (and the corresponding output values of $\sigma_{0.2}$ and $\sigma_{\mathrm{el}}$ ) make up dataset $\mathrm{C}$, the "rule-of-thumb" dataset.

\section{Modelling of the Datasets}

Both the MLR and the NF modelling approaches have been applied to all 3 datasets ( $\mathrm{A}$-raw data, B-microstructure related and C_- "rule-of-thumb").

\subsection{Data Analysis}

Inspection of data distributions, statistical and conditioning diagnostics allowed any collinearity between variables to be identified. Some degree of correlation was found between $x_{\mathrm{Fe}, \mathrm{w}}$ and $x_{\mathrm{Zr}, \mathrm{w}}$ in dataset $\mathrm{A}$ and between $x_{\mathrm{Zn}, \mathrm{w}}: x_{\mathrm{Mg}, \mathrm{w}}$ and $\left(x_{\mathrm{Zn}, \mathrm{w}}+x_{\mathrm{Cu}, \mathrm{w}}\right): x_{\mathrm{Mg}, \mathrm{w}}$ in dataset $C$. Two near linear dependencies were detected in dataset $\mathrm{B}$, the first between $x_{\mathrm{Cu}, \alpha}$ and $x_{\mathrm{S}}$ and the second involving $x_{\mathrm{Mg}, \alpha}, x_{\eta^{\prime}}$ and $x_{\mathrm{Mg}, \mathrm{xs}}$. The collinearities in datasets $B$ and $C$ can be understood directly in terms of the transformations performed in Sec. 4. The correlations in dataset A may be a reflection of the small sample size. Such collinearities are of concern for subsequent regression analyses and inferences, as these data weaknesses will be responsible for high parametric uncertainty and lower confidence in the predictions.

\subsection{MLR Analysis}

Linear regression analysis may be viewed as a transparent approach to modelling, as the inspection of both magnitude and sign of the weights may reveal information about the relative contributions of the inputs. Although insufficiently flexible to perform well in many applications, MLR analyses can help interpreting and understanding the data. Table 1 lists the weights determined for each input variable and the resultant MSE for each dataset for the MLR approach. The MLR approach predicts $\sigma_{0.2}$ with a high MSE with no tangi-
Table 2. Model performance measures for NF modelling of $\sigma_{0.2}$ and $\sigma_{\mathrm{cl}}$ employing three different sets of input data: the raw input data (dataset $A$ ), the set transformed using microstructure related assessments (dataset B) and the set transformed using "rule-of-thumb" sums and ratios (dataset C).

\begin{tabular}{ccccccccccc}
\hline \multicolumn{1}{c}{$\sigma_{0.2}$} & \multicolumn{10}{c}{$\sigma_{\mathrm{cl}}$} \\
\hline Dataset & Model & MSE & LOOCV & SS & $\mathrm{n}_{w}$ & Model & MSE & LOOCV & SS & $\mathrm{n}_{w}$ \\
A & Fig.3 & 451 & 507 & 2394 & 8 & Fig.4 & 0.344 & 0.385 & 1.29 & 5 \\
B & Fig.5 & 544 & 590 & 2444 & 6 & Fig.6 & 0.259 & 0.271 & 0.98 & 5 \\
C & Fig.7 & 358 & 436 & 1898 & 8 & Fig.8 & 0.256 & 0.272 & 1.41 & 7 \\
\hline
\end{tabular}

ble change observed from pre-processing the data (in all cases comparable to an effective standard deviation of error on the prediction of $\pm 25 \mathrm{MPa}$ ). MLR accounted for a similar proportion of the variance in $\sigma_{\mathrm{el}}$ values, again MSE was unaffected by choice of dataset (giving an effective standard deviation of error on the prediction of $\pm 1.85 \%$ IACS). Generally speaking the weights obtained for dataset B appear consistent with the analysis presented in Sec. 4.2. $x_{\eta^{\prime}}$ and $x_{\mathrm{Mg}, \alpha}$ have the largest weights in determining $\sigma_{0.2}$ with the influence of $x_{\mathrm{S}}$ being near to negligible. Increasing ageing time will reduce the strength because the data is obtained mostly for the overaging regime. However the uncertainty in the weights (particularly for collinear variables) is high, and inferences based on individual weights should be made with caution.

\subsection{Neurofuzzy Data Modelling}

The results of the NF model construction algorithms starting from an empty model structure for input datasets A, B and C are presented in Figs. 3-8, whilst model performance measures are listed in Table 2. Comparison of Tables 1 and 2 indicates that NF models yield lower MSE values for predictions of both $\sigma_{0.2}$ and $\sigma_{\text {el }}$. In modelling $\sigma_{0.2}$ from datasets $\mathrm{A}$ and $\mathrm{B}$ a subset of the inputs has been chosen, dataset $\mathrm{C}$ contained the fewest inputs and they have all been retained despite the correlations between the inputs. In all cases an input variable related to $\mathrm{Mg}$ and $t$ have been combined to form a separate subnetwork, leading to the improved MSE over MLR. The other inputs give simple linear trends in behaviour. For all 3 datasets a subset of the inputs has been chosen to model $\sigma_{\mathrm{el}}$ : in all 3 cases the ageing time is modelled by a piecewise linear approximation and this extra flexibility has allowed a considerable improvement in the MSE cf. MLR.

The number of adjustable parameters in the NF model indicates its complexity. Dataset $\mathrm{B}$ generally leads to models with the fewest number of adjustable parameters, which indicates that this transformation of input variables is allowing the identification of a more parsimonious model structure. Dataset B represents the most explicitly descriptive metallurgical information, i.e. it should provide a more transparent feature representation, and the selection of input variables by the NF modelling process should have more relevance to microstructure-property relationships. A better fit is not necessarily an indication of good generalisation perfor- 
(a)

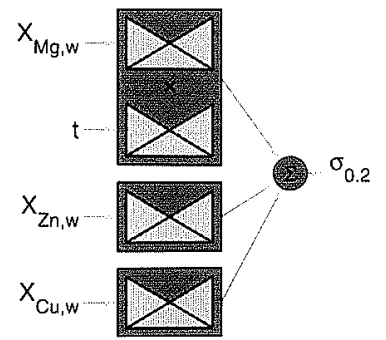

(b)

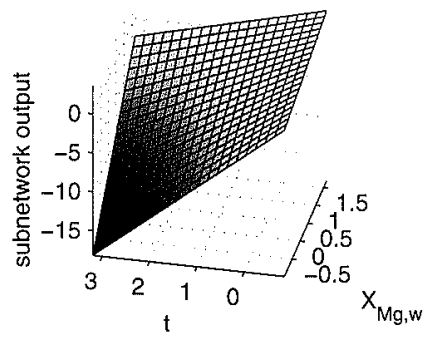

(c)

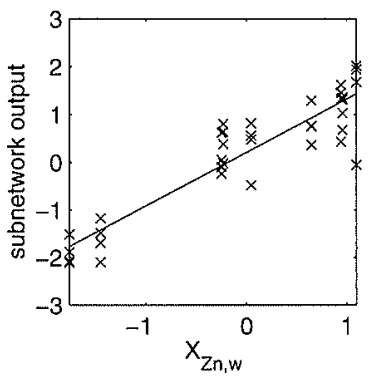

(d)

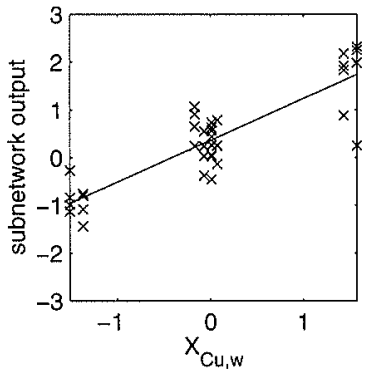

Fig. 3. NF modelling on input dataset $A$ for $\sigma_{0.2}$ : constructed model (a), corresponding subnetwork outputs $v s_{.:} x_{\mathrm{Mg} . \mathrm{w}} \times t(\mathrm{~b}), x_{\mathrm{Zn}, \mathrm{w}}(\mathrm{c})$, and $x_{\mathrm{Cu}, \mathrm{w}}(\mathrm{d})$.

mance. The NF model for dataset B identifies $t$ and $x_{\mathrm{Mg}, \mathrm{xs}}$ as the main variables influencing $\sigma_{\mathrm{el}}$, which is in accordance with the discussion in Sec. 4.2. The selection of $t, x_{\eta^{\prime}}$ and $x_{\mathrm{Mg}, \alpha}$ as the main variables influencing $\sigma_{0.2}$ is again in accordance with Sec. 4.2. One surprising result of the NF model construction for set B is the selection of the tensor product $x_{\mathrm{Mg}, \alpha} \times t$. In terms of microstructure development this indicates that the amount of $\mathrm{Mg}$ dissolved in the Al-rich phase has an important influence on the rate of strength reduction. The latter is due to coarsening of the main phase(s), and it is here suggested that the $\mathrm{Mg} \times t$ dependency may be related to the interaction of $\mathrm{Mg}$ atoms with vacancies, as the latter influences diffusion rates significantly, e.g. ${ }^{28)}$ The $\mathrm{Mg} \times t$ dependency is supported by the fact that for the other two datasets an input variable related to $\mathrm{Mg}$ and $t$ have also been combined to form a tensor product subnetwork structure.

Figures $\mathbf{9}$ and $\mathbf{1 0}$ show the network targets versus network outputs for the NF models corresponding to the three datasets. Training data and test data-based on LOOCV - are shown with error bars representing the uncertainty in the model's output predictions with respect to particular input variable conditions. The ability to derive error bars $^{9)}$ is a valuable property of the model, giving an indication of how well the model performs in different regions of the input space. Test errors are seen to be slightly larger than the training error in all cases and show similar behaviour.

\subsection{Neurofuzzy Model Initialisation}

Unless an exhaustive search for an optimal model is undertaken, the instability of the heuristic model construction algorithms may mean that the model may still settle in a local minima. In this modelling exercise the small sample size has placed a significant restriction on the number of possible adjustable parameters in the model. Initialising the $\sigma_{0.2}$ NF model by including $x_{\mathrm{Cu}, \alpha}$ in addition to the other inputs (i.e. imposing a change in model structure) leads to a largely unchanged SS but 
(a)

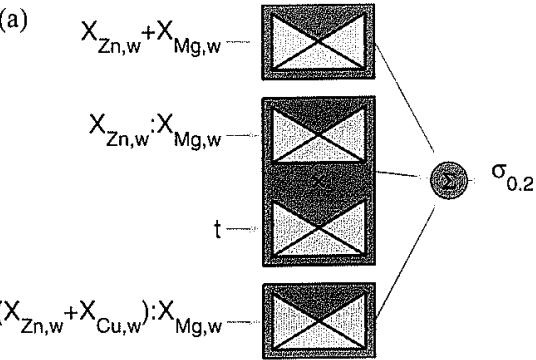

(a)

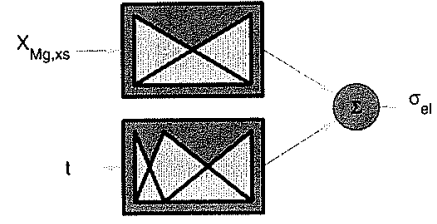

(b)

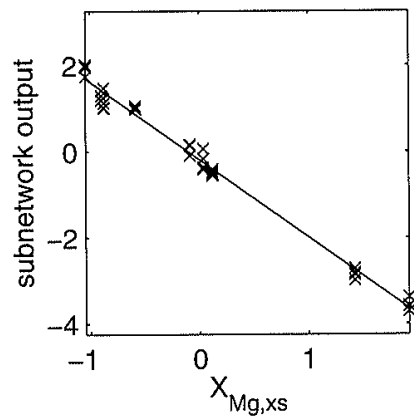

(c)

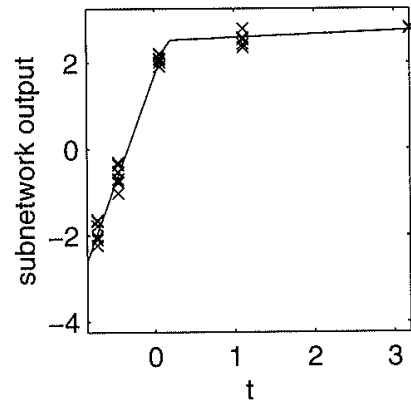

Fig. 6. NF modelling on input dataset $B$ for $\sigma_{\mathrm{cl}}$ : constructed model (a), corresponding subnetwork outputs vs.: $x_{\mathrm{Mg}, \mathrm{xs}}(\mathrm{b})$ and $t(\mathrm{c})$. (b)

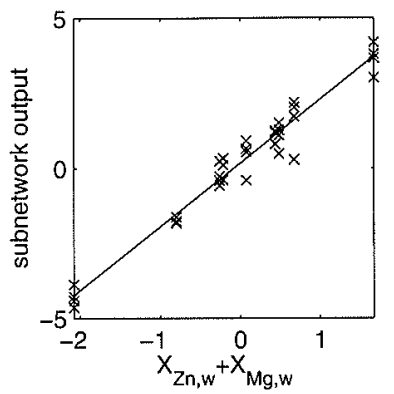

(c)

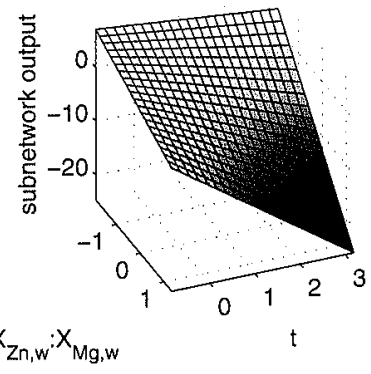

(d)

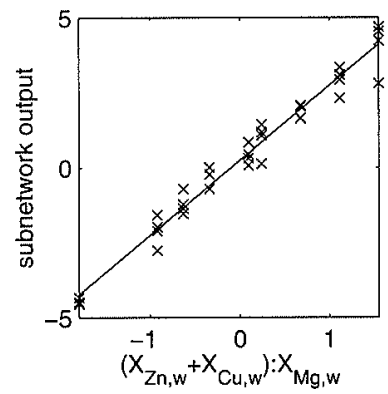

Fig. 7. NF modelling on input dataset $C$ for $\sigma_{0.2}$ : constructed model (a), corresponding subnetwork outputs vs.: $x_{\mathrm{Zn}, \mathrm{w}}+x_{\mathrm{Mg}, \mathrm{w}}(\mathrm{b}), x_{\mathrm{Zn}, \mathrm{w}}$ : $x_{\mathrm{Mg}, \mathrm{w}} \times t(\mathrm{c}),\left(x_{\mathrm{Zn}, \mathrm{w}}+x_{\mathrm{Cu}, \mathrm{w}}\right): x_{\mathrm{Mg}, \mathrm{w}}$ (d). (a)

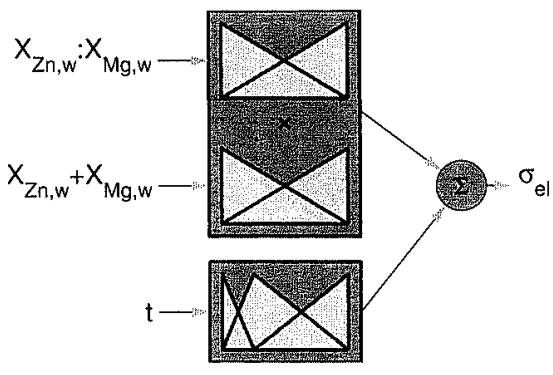

(b)

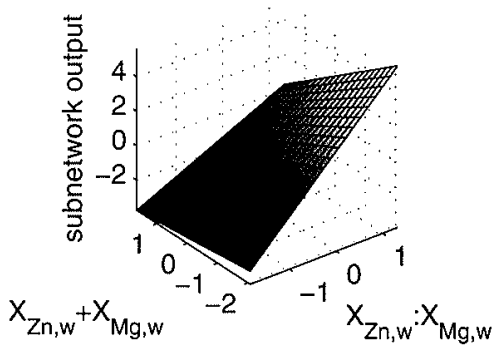

(c)

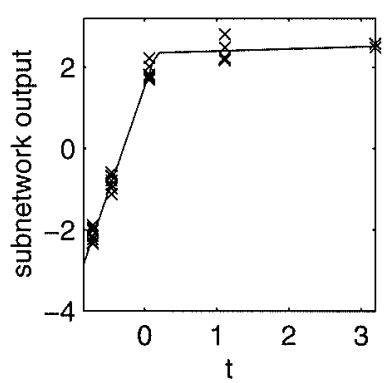

Fig. 8. NF modelling on input dataset $\mathrm{C}$ for $\sigma_{\mathrm{e} 1}$ : constructed model (a), corresponding subnetwork outputs vs.: $\left(x_{\mathrm{Zn}, \mathrm{w}}+x_{\mathrm{Mg}, \mathrm{w}}\right) \times\left(x_{\mathrm{Zn}, \mathrm{w}}\right.$ : $\left.x_{\mathrm{Mg}, \mathrm{w}}\right)(\mathrm{b}), t(\mathrm{c})$. with a much-improved MSE for dataset B. This model structure was identified during the construction of the final model portrayed in Fig. 4(a), but the lack of improvement in SS on including $x_{\mathrm{Cu}, \alpha}$ led to the deletion of this input. In fact, the structure of the $\sigma_{0.2}$ model thus obtained is equivalent to the ones depicted in Fig. 3(a) obtained using dataset $\mathrm{A}$ and in Fig. 7(a) obtained using dataset $\mathrm{C}$, and this model is presented in a general form in Fig. 11. Constraining the model for each dataset to the general form in Fig. 11 provides a comparison of the effect of the data transformations on modelling $\sigma_{0.2}$. Similarly, we may define a general constrained model to compare the effect of data transformations on modelling $\sigma_{\mathrm{el}}$. Comparison of the models in Figs. 4(a), 6(a), and 8(a) shows that the models for $\sigma_{\text {el }}$ are generally given by a summation of a piecewise linear approximation to $t$ with (depending on the input dataset) composition re- lated inputs. Hence, a general model incorporating all these features is the one presented in Fig. 12.

The relative performance of the three different sets of input data when using the initialised model structures (Figs. 11 and 12) is presented in Table 3. In addition to the three sets of input variables (A, B and C) employed thus far we have also included results for a fourth set (set D) which is the same as set B apart from the fact that for the model for $\sigma_{0.2}$ instead of $x_{\mathrm{Mg}, \alpha}, 1 / x_{\mathrm{Mg}, \alpha}$ is taken. This change is not obtained from any microstructural insight, but is based on inspection of some additional NF model constructions of various variable sets which included inputs of the type $x_{\mathrm{Zn}, \mathrm{w}}: x_{\mathrm{Mg}, \mathrm{w}}$, $\left(x_{\mathrm{Zn}, \mathrm{w}}+x_{\mathrm{Cu}, \mathrm{w}}\right): x_{\mathrm{Mg}, \mathrm{w}}, 1 / x_{\mathrm{Mg}, \alpha}$, i.e. variables proportional to $1 / x_{\mathrm{Mg}, \alpha}$ or $1 / x_{\mathrm{Mg}, \mathrm{w}}$, which revealed that this inverse compositional variable yielded improved modelling results. Thus this single modification to set $B$ is based 
(a)

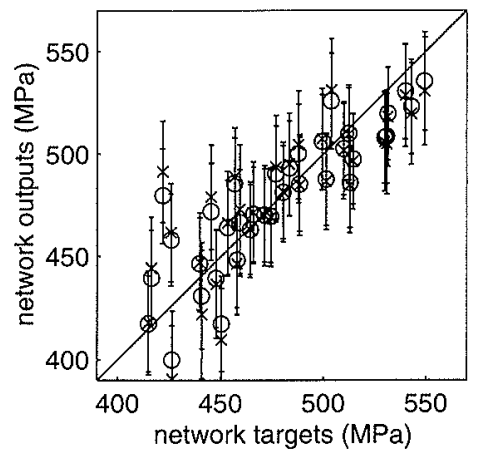

(b)

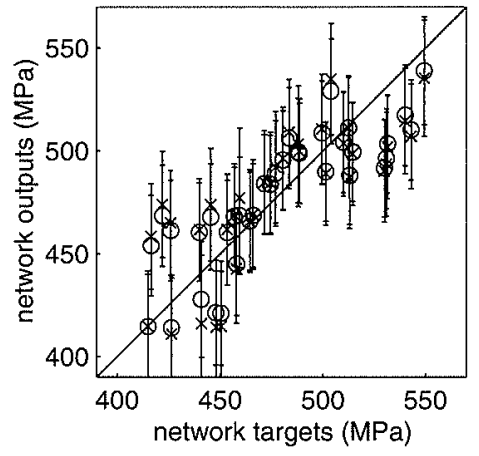

(c)

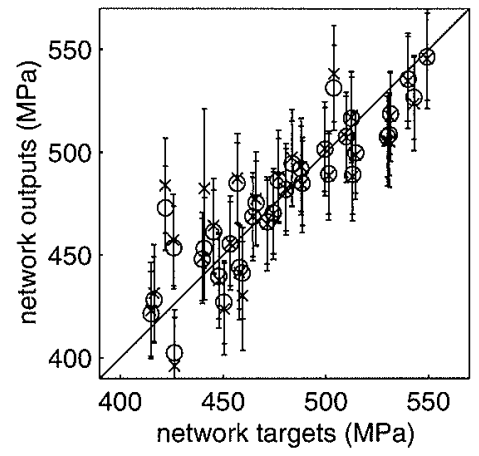

Fig. 9. Prediction scatter plots for the NF models constructed for $\sigma_{0.2}$ from datasets: A (a), B (b) and $C(c)$. Output predictions on both training data $(O)$ and leaveone-out data $(x)$ are shown. (a)

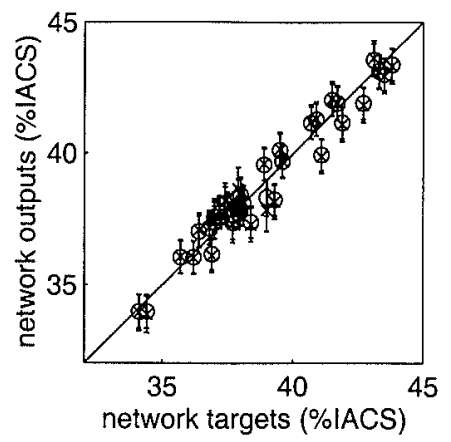

(b)

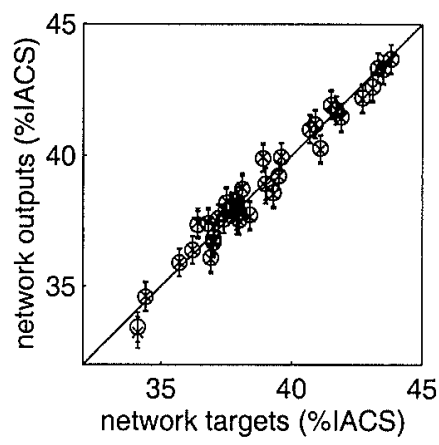

(c)

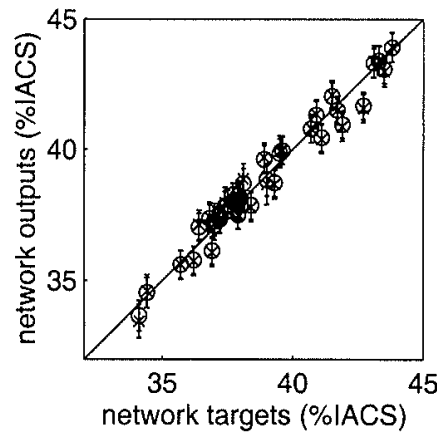

Fig. 10. Prediction scatter plots for the NF models constructed for $\sigma_{\mathrm{e}}$ from datasets: A (a), B (b) and $C$ (c). Output predictions on both training data $(O)$ and leave-one-out data $(x)$ are shown.

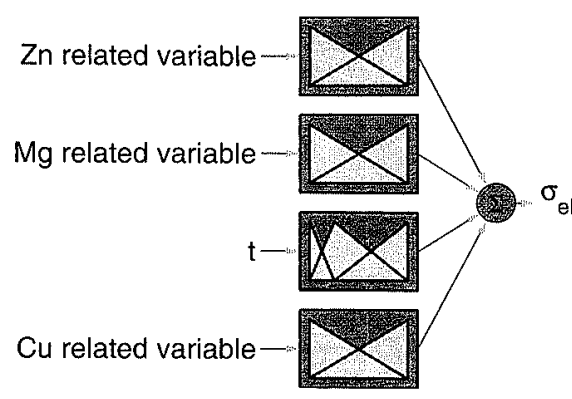

Fig. 11. General structure of the model employed in initialising the NF models for $\sigma_{0.2}$.

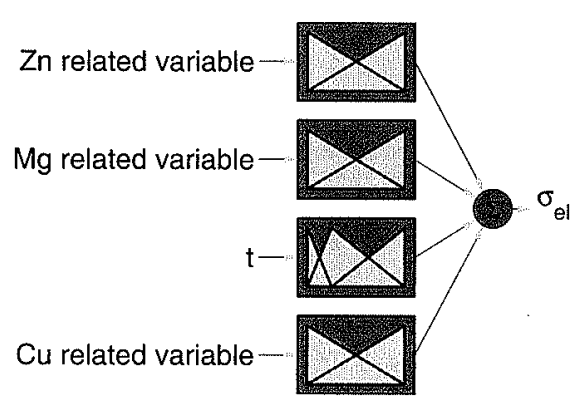

Fig. 12. General structure of the model employed in initialising the NF models for $\sigma_{\mathrm{cl}}$. on NF modelling results inspired by the ratio of variables in set $C$.

\section{Summary and Discussion}

\subsection{Modelling Approaches}

A dataset of $7 x x x$ series-based aluminium alloys with 7 input variables ( 6 alloying element concentrations and ageing time) and 2 output variables (the properties $\sigma_{0.2}$ and $\sigma_{\mathrm{el}}$ ) has been analysed using multiple linear regression (MLR) and NF modelling approaches. For both modelling approaches we evaluated the effectiveness of pre-processing of the input data using 2 types of transformations of the composition variables. In order to facilitate comparisons the root mean square errors (RMSE) on the test data obtained from Tables 1 to 3 are presented graphically in Fig. 13. This shows that both for $\sigma_{0.2}$ and $\sigma_{\text {el }}$ (irrespective of data pre-processing) the NF modelling always yields improved model predictive ability as compared to MLR, with the difference being especially pronounced for $\sigma_{\mathrm{el}}$. These improvements are due to increased flexibility in the models that are constructed in the NF modelling framework which yield a better functional representation than the simple inflexible MLR models. In modelling $\sigma_{0.2}$ the NF approach selects a model which contains a sub-model combining $t$ and a $\mathrm{Mg}$-related parameter $\left(x_{\mathrm{Mg}, \mathrm{w}}\right.$ for set $\mathrm{A}, x_{\mathrm{Mg}, \alpha}$ for set $\mathrm{B}, x_{\mathrm{Zn}, \mathrm{w}}: x_{\mathrm{Mg}, \mathrm{w}}$ for set $\mathrm{C}$, and $1 / x_{\mathrm{Mg}, \alpha}$ for set D), and for the modelling of $\sigma_{\mathrm{el}}$ the NF approach selects a model which contains a piecewise linear approximation for the $t$-dependency. The reduced SS and MSE values indicate that these refinements have led to better modelling performance. This indicates a model structure that is supported by the available data.

In comparing the modelling performance obtained using the various input datasets $(A, B$ and $C$ ) a mixed picture emerges, and prediction of $\sigma_{0.2}$ and $\sigma_{\mathrm{el}}$ have to 
Table 3. Training set MSE and LOOCV prediction MSE estimates obtained for the initialised models for $\sigma_{0.2}$ and $\sigma_{e 1}$ for three different sets of input data: the raw input data (dataset $\mathrm{A}$ ), the set transformed using microstructure related assessments (dataset $B$ ) and the set transformed using "rule-of-thumb" sums and ratios (dataset $\mathrm{C}$ ) and the $1 / x_{\mathrm{Mg}, x}$ (dataset D).

\begin{tabular}{|c|c|c|c|c|c|c|}
\hline Dataset & $\sigma_{0.2}$ & & & $\sigma_{\mathrm{el}}$ & & \\
\hline & Variables & MSE & LOOCV & variables & MSE & LOOCV \\
\hline $\begin{array}{l}\text { A: no } \\
\text { transformation } \\
\text { of inputs }\end{array}$ & 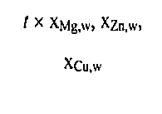 & 451 & 507 & $\begin{array}{c}t, \mathrm{x}_{\mathrm{Mz}_{\mathrm{w}, w},} \mathrm{x}_{\mathrm{Zn,w},} \\
\mathrm{x}_{\mathrm{Cu}_{\mathrm{u}, w}}\end{array}$ & 0.279 & 0.291 \\
\hline $\begin{array}{l}\text { B: } \\
\text { microstructure } \\
\text { related data }\end{array}$ & $t \times \mathrm{x}_{\mathrm{Mg}, \alpha,}, \mathrm{x}_{\eta}, \mathrm{x}_{\mathrm{Cu}, a}$ & 468 & 528 & $t, x_{M g_{B}, s_{2}} x_{\eta^{\prime}}, x_{C_{\mu, \alpha}}$ & 0.204 & 0.219 \\
\hline $\begin{array}{l}\text { C: 'rule of } \\
\text { thumb' based } \\
\text { ratios }\end{array}$ & $\begin{array}{c}t \times x_{Z_{n, w}:} x_{M g, w} \\
x_{Z_{n, w},}+x_{M g, w,} \\
\left(x_{Z_{n, w},}+x_{C l, w}\right): x_{M_{g, w}}\end{array}$ & 358 & 436 & 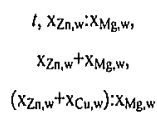 & 0.396 & 0.452 \\
\hline $\begin{array}{l}\text { D: approach B } \\
\text { taking } 1 / x_{M g, \alpha}\end{array}$ & $\begin{array}{c}I \times 1 / \mathrm{x}_{\mathrm{Mg}_{\mathrm{g}, \alpha}}, \mathrm{x}_{\mathrm{r}^{\prime}}, \\
\mathrm{x}_{\mathrm{C}_{\mathrm{C}, Q},}\end{array}$ & 411 & 466 & & & \\
\hline
\end{tabular}

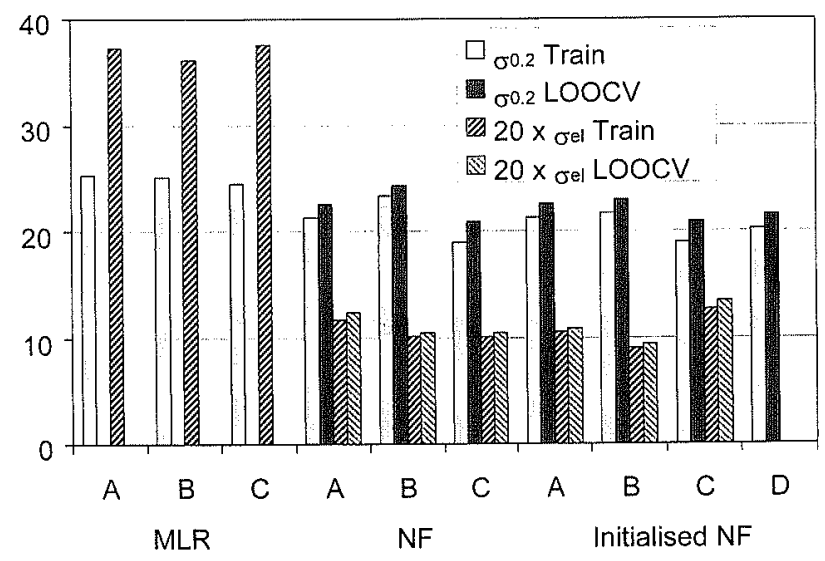

Fig. 13. Comparison between training and LOOCV estimates of RMSE for the modelling approaches pursued (MLR, NF, initialised NF models) and data preprocessing $(A, B, C)$.

be discussed separately. For modelling $\sigma_{\mathrm{cl}}$, Fig. 13 shows that the use of transformed input dataset $B$ is clearly beneficial, and it is concluded that dataset $\mathrm{B}$ has represented the data in explicit microstructural features so that the specific effects of different microstructure contributions can be assessed. In contrast, for modelling $\sigma_{0.2}$, Fig. 13 shows that the use of transformed input dataset $\mathrm{C}$ is clearly the most beneficial. Notwithstanding the better performance of dataset $\mathrm{C}$ in modelling the yield strength data, there are still a number of benefits to using dataset $B$ in model construction. Firstly, with dataset B a more parsimonious (fewest number of adjustable parameters) model can be identified (e.g. the model in Fig. 5, which contains just three inputs), thus enabling a better insight to the relevant physical parameters and avoiding overfitting. Secondly, models using dataset B are likely to yield a better generalisation performance, as this dataset has benefitted from the inclusion of a priori knowledge. For example, it will be more accurate in extrapolating beyond the current composition range. This can be illustrated from a detailed analysis of the dependency of yield strength on the $\mathrm{Mg}$ content of the alloys (not presented here), which shows that models obtained with dataset $C$ will, unrealistically, predict a single stage, monotonically increasing yield strength with increasing $\mathrm{Mg}$ content, whilst models obtained with dataset $\mathrm{B}$ will predict a (more realistic) levelling off of the strength increase obtainable by increasing the $\mathrm{Mg}$ content.

It is further noted that the modelling using dataset $D$ reveals that the relatively weaker performance (in terms of MSE and LOOCV in Table 3) of dataset B as compared to dataset $C$ in modelling of the yield strength is mainly due to the construction of the sub-model including a $\mathrm{Mg}$ related parameter and $t$, i.e. it is related to the way in which $\mathrm{Mg}$ influences the kinetics of the reaction and does not directly indicate that the simplified ideas concerning the strengthening mechanisms (Sec. 4.2) are inaccurate.

The modelling cycle identified in the present work consists of:

(1) data inspection/understanding $\leftrightarrow$ (2) dataset selection $\leftrightarrow(3)$ empirical modelling (model construction) $\leftrightarrow(4)$ model validation (physical insight) $\leftrightarrow(1)$ etc.

Based on inspection of the FS/BE model constructions, general initialised models were defined for each property with a similar structure (Figs. 11 and 12) for each dataset. This formed a basis for comparison of the effects on modelling performance for each dataset transformation. In modelling $\sigma_{0.2}$ the initialised model for dataset $\mathrm{C}$ showed approximately a $10 \%$ reduction in the test RMSE cf. test RMSEs for datasets A and B; dataset $\mathrm{D}$-produced as a result of the modelling cycle defined earlier and is a combination of physically based transformations (dataset B) and a transformation suggested by inspection of NF model constructions-exhibited an improved test RMSE over dataset B. In modelling $\sigma_{\mathrm{el}}$, the initialised model for dataset B showed at least a $15 \%$ reduction in test RMSE cf. test RMSEs over datasets $\mathrm{A}$ and $\mathrm{C}$.

\subsection{Microstructure-Property Relations of $7 x x x$ Alloys}

The present analysis can be used to draw out some of the microstructure property issues for the present, complex $7 x x x$ (Al-Zn-Mg-Cu) alloys. Firstly it is noted that compared to the original untransformed dataset (A) the dataset transformed using some relatively simple information on the microstructure (B) yielded a considerable improvement in NF modelling performance for $\sigma_{\mathrm{e} 1}$ but no improvement in the modelling of $\sigma_{0.2}$. In retrospect this difference is not surprising as strength is the more complex property, more dependent on additional microstructural features that are not directly included in the available inputs (e.g. grain size, precipitate size distributions, precipitate free zones). Both the MLR and the NF modelling confirm the main expected structure-property relationships, for example, the maximum amount of $\eta^{\prime}, x_{\eta^{\prime}}$, is important in determining the strength (see MLR results in Table 1 and results of NF model construction in Fig. 5). In addition, the NF approach revealed that for $\sigma_{0.2}$, a sub-model of $t$ and $\mathrm{Mg}$ concentration improves modelling performance statistics. (Note that in the course of the NF iterative model search, 
a network structure encompassing two univariate additive sub-models-one for $t$ and one for $\mathrm{Mg}$ - was considered, and rejected in favour of the sub-model of $t$ and Mg.) It is suggested that this sub-model in essence represents the complex interaction of dissolved $\mathrm{Mg}$ with vacancies, which will influence the rate of ageing: possibly vacancies bind to the $\mathrm{Mg}$ atoms enhancing ageing in the present overaged alloys.

At present the modelling of the properties of $7 x x x$ alloys on the basis of microstructural knowledge is further pursued by combining kinetic equations (e.g. Johnson-Mehl-Avrami-Kolmogorov ${ }^{29,30)}$ or StarinkZahra ${ }^{31-33)}$ type equations), coarsening models and microstructural investigation. Initial results are promising: further enhancement of the accuracy of model predictions is possible using this approach, where prespecified regression functions (based on sound physical metallurgy) are being used. This, however, does not detract from the data-driven results reported in the present paper. NF modelling, combined with suitable transformation of input data and model initialisation, is a transparent approach which allows us to gain information about relationships in the data through the modelling process and provides a route by which purely empirical modelling approaches can be combined with physical modelling relatively easily. Such an approach requires only limited knowledge of the system investigated, and is hence an important tool for analysis of complicated materials processing issues.

\section{Conclusions}

In considering the overall implications of the modelling approaches attempted in the present paper it is clear that the combination of NF approaches to model selection/construction combined with transformation of the input data has been successful in improving the modelling performance for the present $7 x x x$ Al-alloy dataset. In the first iteration, an optimal feature extraction for models constructed by the NF approach was obtained using input dataset $\mathrm{B}$ based on microstructural data (precipitation sequence, precipitate compositions) to model $\sigma_{\mathrm{el}}$ and dataset $\mathrm{C}$ based on "ruleof-thumb" ratios and sums to model $\sigma_{0.2}$. In a second iteration, inspection of NF models resulted in a minor change in dataset $\mathrm{B}$ that yielded dataset $\mathrm{D}$, which proved to be the most successful one in overall terms. A modelling cycle is identified whereby (1) data inspection/understanding $\leftrightarrow$ (2) dataset selection $\leftrightarrow$ (3) empirical modelling (model construction) $\leftrightarrow(4)$ model validation (physical insight $) \leftrightarrow(1)$ etc. The use of empirical modelling approaches as an assessment of underlying structures in the data is proposed as an essential part of modelling based on well-founded physically based relationships. In situations where more limited physical understanding exists, a NF modelling approach offers a combination of pure empirical modelling and physically based modelling possibilities.

\section{Acknowledgements}

Thanks are due to Dr. P. D. Pitcher at MSS, DERA and Dr. J. Newman, British Aluminium Plate for provision of the dataset, financial support from Federal Mogul and the University of Southampton is also gratefully acknowledged.

\section{REFERENCES}

1) M. Brown and C. J. Harris: Neurofuzzy Adaptive Modelling and Control, Prentice Hall, Hemel Hempsted (1994).

2) C. J. Harris, M. Brown, K. M. Bossley, D. J. Mills and M. Feng: J. Eng. Apps, AI, 9 (1996), 1.

3) J. M. Schooling, M. Brown and P. A. S. Reed: Mater. Sci. Eng. $A, 260$ (1999), 222.

4) J. M. Schooling and P. A. S. Reed: Proc. of 8th Int. TMS Conf on Superalloys, TMS, Warrendale, PA, (1996), 409.

5) A. Mukherjee, S. Schmauder and M. Ruhle: Acta Metall Mater., 43 (1995), 4083

6) H. K. D. H. Bhadeshia, D. J. C. MacKay and L.-E. Svensson: Mat. Sci. Tech., 11 (1995), 1046.

7) L. Gavard, H. K. D. H. Bhadeshia, D. J. C. MacKay and S. Suzuki: Mat. Sci. Tech., 12 (1996), 453.

8) M. Brown and C. J. Harris: Int. J. of Neural Syst., 6 (1995), 2, 197.

9) K. M. Bossley: Neurofuzzy modelling approaches in system identification, Ph.D. thesis, Southampton, (1997).

10) M. Brown, K. M. Bossley and C. J. Harris: EUFIT '96, Vol. 2, (1996), 762.

11) S. R. Gunn, M. Brown and K. M. Bossley: Lecture Notes in Computer Science n. 1208, Springer-Verlag, Berlin, (1997), 313.

12) V. N. Vapnik: The Nature of Statistical Learning Theory. Springer-Verlag, New York, (1995).

13) R. H. Brown and L. A. Willey: in Aluminium: Properties, Physical Metallurgy and Phase Diagrams, ed. by K. R. van Horn, ASM, Ohio, (1967)), 31.

14) L. F. Mondolfo: Aluminium Alloys, Butterworths \& Co Ltd, London, (1976), 652.

15) P. Villars, A. Prince and H. Okamoto: ASM Handbook of Ternary Alloy Diagrams, ASM International, Materials Park, Ohio, (1995).

16) D. J. Strawbridge, W. Hume-Rothery and A. T. Little: J. Inst. Metals, 74 (1948), 191.

17) H. Liang, S.-L. Chen and Y.A. Chang: Metall. Mater. Trans. A, 28 (1997), 1725.

18) P. Liang, T. Tarfa, J. A. Robinson, S. Wagner, P. Ochin, M. G. Harmelin, H. J. Seifert, H. L. Lukas and F. Aldinger: Thermochim. Acta, 314 (1998), 87

19) P. Sainfort, C. Sigli, G. M. Raynaud and Ph. Gomiero: Mater. Sci. Forum, 242 (1997), 25.

20) T. J. Warner, R. A. Shahani, P. Lassince and G. M. Raynaud: 3rd ASM Conf. on Synthesis, Processing and Modelling of Advanced Materials, Paris, (1997).

21) A. Deschamps, F. Livet and Y. Brechet: Acta Mater., 47 (1999), 293.

22) M. J. Starink and P. J. Gregson: Scr. Metall. Mater., 33 (1995), 893.

23) M. J. Starink and P. J. Gregson: Mater. Sci. Forum, 217-222 (1996), 673

24) J. K. Park and A. J. Ardell: Scr. Metall., 22 (1988), 1115.

25) P. Gomiero, Y. Brechet, F. Louchet, A. Tourabi and B. Wack: Acta Metall. Mater., 40 (1992), 857.

26) M. J. Starink, P. Wang, I. Sinclair and P. J. Gregson: Acta Mater., in press

27) K. R. Anderson: US Patent no. 5312498 (1994).

28) M. J. Starink and A.-M. Zahra: Acta Mater., 46 (1998), 3381.

29) J. W. Christian: The Theory of Transformation in Metals and Alloys, 2nd ed., Part 1, Pergamon Press, Oxford, (1975)

30) V. Sessa, M. Fanfoni and M. Tomellini: Phys. Rev. B, 54 (1996), 836.

31) M. J. Starink and A.-M. Zahra: Thermochim. Acta, 292 (1997), 159

32) M. J. Starink and A.-M. Zahra: Acta Mater., 46 (1998) 3381.

33) M. J. Starink and A.-M. Zahra: Phil. Mag., A77 (1998) 187. 